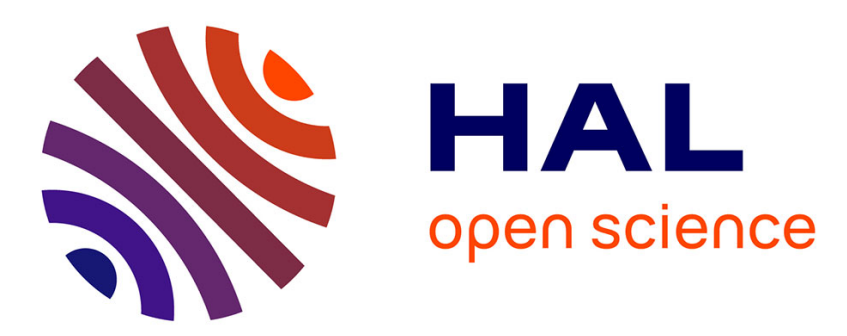

\title{
Hierarchical Laplacian and Its Spectrum in Ultrametric Image Processing \\ Jesus Angulo
}

\section{To cite this version:}

Jesus Angulo. Hierarchical Laplacian and Its Spectrum in Ultrametric Image Processing. International Symposium on Mathematical Morphology and Its Applications to Signal and Image Processing ISMM 2019, Jul 2019, Saarbrücken, Germany. pp.29-40, 10.1007/978-3-030-20867-7_3 . hal-02436474

\section{HAL Id: hal-02436474 https://hal.science/hal-02436474}

Submitted on 13 Jan 2020

HAL is a multi-disciplinary open access archive for the deposit and dissemination of scientific research documents, whether they are published or not. The documents may come from teaching and research institutions in France or abroad, or from public or private research centers.
L'archive ouverte pluridisciplinaire HAL, est destinée au dépôt et à la diffusion de documents scientifiques de niveau recherche, publiés ou non, émanant des établissements d'enseignement et de recherche français ou étrangers, des laboratoires publics ou privés. 


\title{
Hierarchical Laplacian and its Spectrum in Ultrametric Image Processing
}

\author{
Jesús Angulo \\ MINES ParisTech, PSL-Research University, \\ CMM-Centre de Morphologie Mathématique, France \\ jesus. angulo@mines-paristech.fr
}

\begin{abstract}
The Laplacian of an image is one of the simplest and useful image processing tools which highlights regions of rapid intensity change and therefore it is applied for edge detection and contrast enhancement. This paper deals with the definition of the Laplacian operator on ultrametric spaces as well as its spectral representation in terms of the corresponding eigenfunctions and eigenvalues. The theory reviewed here provides the computational framework to process images or signals defined on a hierarchical representation associated to an ultrametric space. In particular, image regularization by ultrametric heat kernel filtering and image enhancement by hierarchical Laplacian are illustrated.
\end{abstract}

Keywords: ultrametric space; semigroups on ultrametric spaces; hierarchical Laplacian; ultrametric image processing;

\section{Introduction}

The Laplace operator (or Laplacian) of an image is one of the simplest and useful image processing tools, since it highlights regions of rapid intensity change and therefore it is applied for edge detection (zero crossing edge detector [9]) and contrast enhancement by subtraction from the original image.

The Laplace operator is defined as the divergence of the gradient. When applied to a function $f$ on $\mathbb{R}^{n}$, the Laplacian produces a scalar function given by $L(f)=\nabla \circ \nabla f=\Delta f=\sum_{i=1}^{n} \frac{\partial^{2} f}{\partial x_{i}^{2}}$. The second order differentiation becomes in the discrete counterpart a second order difference; i.e., in 1D, $f^{\prime}(x)=$ $(f(x+h / 2)-f(x-h / 2)) / h$ and $f^{\prime \prime}(x)=(f(x+h)+f(x-h)-2 f(x)) / h^{2}$. Hence, the discrete Laplace operator of an image $f$ is given by $L(f)(x)=$ $\sum_{z \in \tilde{N}(x)} f(z)-|\widetilde{N}| f(x)$, where $\widetilde{N}(x)$ is the local neighbourhood of pixel $x$, excluding itself. This transformation can be calculated using a convolution filter with the corresponding kernels. The Laplace operator is also naturally defined on Riemanian manifolds, the so-called Laplace-Beltrami operator, which has been also widely used in image and shape processing [11]. In the litterature of mathematical morphology, the notion of morphological (flat) Laplacian is defined as a second-order operator given by the difference between the gradient by dilation and the gradient by erosion [10], and can be interpreted just as the $\infty$-Laplace operator [8]. 
The goal of this paper is to consider the definition of the Laplacian operator on ultrametric spaces as well as its spectral representation in terms of the corresponding eigenfunctions and eigenvalues. Indeed, the theory of the heat semigroup and Laplacian operator in the case of ultrametric spaces has been developed in a series of papers by Bendikov and collaborators [2,3,4]. Our purpose here is just to review the main results of this theory and to bring it to the context of ultrametric image processing.

Let us precise that ultrametric image or data processing means in our context. The image domain can be considered as an ultrametric space, where the pixels/vertices of the image are hierarchically organized into clusters at different aggregation levels. Interaction between image pixels or vertices is associated to the ultrametric distance. This kind of representation is naturally used in image segmentation, in the case of morphological image segmentation, hierarchical representations are ubiquitous $[12,13,6]$, and the algorithmic ingredients to construct them are typically minimum spanning trees and quasi-flat zones. Once an image has been endowed with a hierarchical structure (i.e., the image domain is an ultrametric space), the image can be not only segmented, but also filtered out, enhanced and so on, according to such representation. In this context, we have introduced the corresponding ultrametric morphological semigroups and scalespaces [1]. This paper is a step forwards in the program of revisiting classical image/data processing on ultrametric representations.

The rest of the paper is organized as follows. Section 2 provides some background definitions on ultrametric spaces. The notion of heat semigroup on ultrametric spaces is briefly reminded in Section 3, which is required to review the notion of Laplacian on an ultrametric space in Section 4. Section 5 considers in particular the case of the Laplacian on a discrete ultrametric space, which is the relevant case for ultrametric image processing. Some illustrative examples of ultrametric image Laplacian are discussed in Section 6. Finally, Section 7 closes the paper with some conclusions and perspectives.

\section{Preliminaries}

Ultrametric space. Let $(X, d)$ be a metric space. The metric $d$ is called an ultrametric if it satisfies the ultrametric inequality, i.e., $d(x, y) \leq \max \{d(x, z), d(z, y)\}$, that is obviously stronger than the usual triangle inequality. In this case $(X, d)$ is called an ultrametric space.

Consider the metric balls $B_{r}(x)=\{y \in X: d(x, y) \leq r\}$. The ultrametric inequality implies that any two metric balls are either disjoint or one contains the other. In particular, every two balls of the same radius are disjoint or coincide. Thus, every point inside a ball is its center. As a consequence of these properties, the collection of all distinct balls of the same radius $r$ forms a partition $X$; for increasing values of $r$, the balls are also increasing, hence we obtain a family of nested partitions of $X$ which forms a hierarchy. This is a fundamental property of ultrametric spaces. 
Throughout the rest of the paper, we consider the triplet $(X, d, \mu)$, where $(X, d)$ is a compact separable ultrametric space; compactness involves that all balls are compact and separability that the set of all values of metric $d$ is at most countable and all distinct balls of a given radius $r>0$ form at most a countable partition of $X$. The measure $\mu$ is a Radom measure on $X$ with full support, such that $0<\mu\left(B_{r}(x)\right)<\infty$ for all $x \in X$ and $r>0$.

Discrete ultrametric space. An ultrametric space $(X, d)$ is called discrete if : i) the set $X$ is countable, ii) all ultrametric balls $B_{r}(x)$ are finite, and iii) the distance function $d$ takes only integer numbers. Given a discrete ultrametric space $(X, d)$, one can consider any measure $\mu$ on $2^{X}$ such that $0<\mu(x)<\infty$ for any $x \in X$ and $\mu(X)=\infty$. For example, $\mu$ can be a counting measure.

Set of ultrametric balls $\mathcal{B}$. Denote by $\mathcal{B}$ be the family (countable set) of all balls $C$ in $X$ with positive radii, and thus positive measure $\mu(C)>0$. Using the hierarchical interpretation of ultrametric balls, for any ball $C \in \mathcal{B}$ such that $C \neq X$ there is a unique parent (or predecessor) ball $B$ which contains $C$. In this case, $C$ is called the child (or successor) of $B$. For any ball $B$ with diam $(B)>0$ the number $\operatorname{deg}(B)$ of its children satisfies $2 \leq \operatorname{deg}(B)<\infty$. Moreover, all the children of $B$ are disjoint and their union is equal to $B$. Let us denote by $C \prec B$ that $C$ is a child of $B$. The functions $\left\{\mathbf{1}_{C}: C \prec B\right\}$ are linearly independent (i.e., orthogonal) and therefore we have

$$
\left\langle\mathbf{1}_{C}, \mathbf{1}_{C^{\prime}}\right\rangle=0, C \prec B, C^{\prime} \prec B \text {, and } \sum_{C \prec B} \mathbf{1}_{C}=\mathbf{1}_{B} .
$$

Intrinsic ultrametric $d_{*}$. Let us first note that different ultrametric distances on $X$ can produce the same set $\mathcal{B}$. In the case of $(X, d)$, for any $x, y \in X$, we denote $B(x \curlywedge y)$ the minimal ball containing both $x$ and $y$. The intrinsic ultrametric is defined as: $d_{*}(x, y)=\operatorname{diam}(B(x \curlywedge y))$ when $x \neq y$ and $d_{*}(x, y)=0$ when $x=y$. Note that the ultrametrics $d$ and $d_{*}$ generates the same set of balls, with a feasible change of their diameter function.

\section{Heat semigroup and heat kernel on ultrametric spaces}

We review in this section some background material on ultrametric heat semigroups from $[2,3]$. The first operator, the averaging one, is the building block for the ultrametic heat kernel theory.

Averaging operator $Q_{r}$. Let us define the family $\left\{\mathrm{Q}_{r}\right\}_{r>0}$ of averaging operators acting on non-negative (or bounded) Borel functions $f: X \rightarrow \mathbb{R}$ by

$$
\mathrm{Q}_{r} f(x)=\frac{1}{\mu\left(B_{r}(x)\right)} \int_{B_{r}(x)} f d \mu
$$

As we can see, that corresponds to computing the mean value at each class of the partition of radius $r$ of the hierarchy associated to $d_{*}$. It is symmetric in $x, y$ 
because $B_{r}(x)=B_{r}(y)$ for any $y \in B_{r}(x)$. We set $\mathrm{Q}_{0}:=$ id. If $\mu(X)=\infty$ then $\mathrm{Q}_{\infty}=0$, while in the case $\mu(X)<\infty$ we have $\mathrm{Q}_{\infty} f=\frac{1}{\mu(X)} \int_{X} f d \mu$. We have $\mathrm{Q}_{r} f \geq 0$ if $f \geq 0$ and $\mathrm{Q}_{r} 1=1$.

$\mathrm{Q}_{r}$ is a Markov operator on the space of bounded Borel functions on $L^{2}(X, \mu)$, which satisfies the following (supremal) semigroup property [3]:

$$
\mathrm{Q}_{r} \mathrm{Q}_{s}=\mathrm{Q}_{s} \mathrm{Q}_{r}=\mathrm{Q}_{\max \{r, s\}} \text {, with } \mathrm{Q}_{r} \mathrm{Q}_{r} f=\mathrm{Q}_{r} f \text {. }
$$

Ultrametric heat semigroup $\left\{P_{t} f\right\}_{t>0}$. Let us choose a distance probability distribution function $\sigma$ that satisfies the following assumptions: $\sigma:[0, \infty] \rightarrow[0,1]$ is a strictly monotone increasing left-continuous function such that $\sigma(0+)=0$ and $\sigma(\infty)=1$. Typically, $\sigma(r)=\exp (-1 / r)$. The operator $P$ determined by the triple $(d, \mu, \sigma)$

$$
P f=\int_{0}^{\infty} \mathrm{Q}_{r} f d \sigma(r)
$$

is an isotropic Markov operator which determines a discrete time Markov chain $\left\{\mathcal{X}_{n}\right\}_{n \in \mathbb{N}}$ on $X$ with the following transition rule: $\mathcal{X}_{n+1}$ is $\mu$-uniformly distributed in $B_{r}\left(\mathcal{X}_{n}\right)$ where the radius $r$ is chosen at random according to the probability distribution $\sigma$.

The Markov operator $P$ is non-negative definite, which allows us to define $P_{t}$ for all $t \geq 0$ using the power $t$ of distribution function $\sigma$. Then $\left\{P_{t}\right\}_{t>0}$ is a symmetric strongly continuous Markov semigroup where $P_{t}$ admits for $t>0$ the following representation:

$$
P_{t} f(x)=\int_{0}^{\infty} \mathrm{Q}_{r} f(x) d \sigma^{t}(r),
$$

which satisfies the following (additive) semigroup property

$$
P_{s} P_{t}=P_{s+t} \text {. }
$$

The family $\left\{P_{t}\right\}_{t \geq 0}$ is a strongly continuous symmetric Markov semigroup on $L^{2}(X, \mu)$.

Ultrametric heat kernel $p_{t}(x, y)$. For any $t>0$, the operator $P_{t}$ admits an integral kernel $p_{t}(x, y)$, i.e., for all $f \in \mathcal{B}_{b}$ and $f \in L^{2}$, one has

$$
P_{t} f(x)=\int_{X} p_{t}(x, y) f(y) d \mu(y),
$$

where $p_{t}(x, y)$ is given by

$$
p_{t}(x, y)=\int_{[d(x, y), \infty)} \frac{d \sigma^{t}(r)}{\mu\left(B_{r}(x)\right)} .
$$

The function $p_{t}(x, y)$ is called the heat kernel of the semigroup $\left\{P^{t}\right\}$. For all $x, y \in X$ and $t>0$, we have: i) $p_{t}(x, y)>0$; ii) $p_{t}(x, y)=p_{t}(y, x)$; iii) $p_{t}(x, y) \leq$ 
$\min \left\{p_{t}(x, x), p_{t}(y, y)\right\}$. The function $(x, y) \mapsto \frac{1}{p_{t}(x, y)}$ if $x \neq y$ and 0 if $x=y$ is an ultrametric.

Let us introduce the intrinsic ultrametric distance associated to $(d, \mu, \sigma)$ :

$$
d_{*}(x, y)=[-\log \sigma(d(x, y))]^{-1} .
$$

For any $r \geq 0$, set

$$
r \mapsto s=[-\log \sigma(r)]^{-1} .
$$

Using the fact that $\sigma$ is strictly monotone increasing, the following identity holds $\forall x \in X: B_{s}^{*}(x)=B_{r}(x)$. Consequently, the metrics $d$ and $d_{*}$ determine the same set of balls and the same topology.

Heat kernel and heat semigroup in ultrametric discrete space. For practical applications and in particular, in ultrametric image processing, we deal with discrete spaces. In such a framework, the computation of the heat semigroup becomes very efficient since the integral of ultrametric distance becomes a weighted sum of averaging operators. The latter only involves an operator at each level of the hierarchy.

Let $(X, d, \mu)$ be a discrete ultrametric space with a counting mesaure $\mu$ on $2^{X}$. The idea now is to replace the ultrametric distance distribution fucntion $\sigma$ by a discrete distribution of weighting values in $(0,1]$. Namely, choose a sequence $\left\{c_{k}\right\}_{k=0}^{\infty}$ of strictly positive reals such that $\sum_{k=0}^{\infty} c_{k}=1$. Let us introduce $s_{k}=$ $\sum_{i=0}^{k} c_{i}$ such that $0<s_{k-1}<s_{k}<1, k=0,1, \cdots$ and $s_{k} \rightarrow 1$ as $k \rightarrow \infty$; $s_{-1}=0$.

Using the distribution of weights $\left\{s_{k}\right\}_{k=0}^{\infty}$, the ultrametric averaging operator, heat semigroup and heat kernel acting on function $f$ are respectively given:

$$
\begin{aligned}
\mathrm{Q}_{k} f(x) & =\frac{1}{\mu\left(B_{k}(x)\right)} \sum_{y \in B_{k}(x)} f(y) ; \\
P_{t} f(x) & =\sum_{k=0}^{\infty} s_{k}^{t}\left(\mathrm{Q}_{k} f(x)-\mathrm{Q}_{k+1} f(x)\right)=\sum_{k=0}^{\infty}\left(s_{k}^{t}-s_{k-1}^{t}\right) \mathrm{Q}_{k} f(x) ; \\
p_{t}(x, y) & =\sum_{k=d(x, y)}^{\infty}\left(s_{k}^{t}-s_{k-1}^{t}\right) \frac{1}{\mu\left(B_{k}(x)\right)} .
\end{aligned}
$$

Note that the second equality in (8) is based on the Abel transformation (i.e., summation by parts of sequences) [2]. Since $\left\{s_{k}^{t}-s_{k-1}^{t}\right\}_{k=0}^{\infty}, t>0$, is a stochastic sequence, the operator $P_{t}$ is Markov. The semigroup indentity here $P_{t} P_{s}=P_{t+s}$ follows from the functional calculus.

The equivalence of the continuous semigroup (5) and the discrete counterpart (8) is obtained by taking density function: $\sigma_{k}=1-s_{k}=\sum_{l>k} c_{l}, k \geq 0$. Clearly $\left\{\sigma_{k}\right\}_{k=0}^{\infty}$ can be any sequence of positive real that satisfies the conditions: $\sigma_{k+1}<\sigma_{k}<1, k=0,1, \cdots$, and $\sigma_{k} \rightarrow 0$ as $k \rightarrow \infty$. 


\section{Laplacian on an ultrametric space}

Laplace operator as a spectral decomposition on $(d, \mu, \sigma)$. Let the spectral resolution $\left\{E_{\lambda}\right\}$ be defined as:

$$
E_{\lambda}=\left\{\begin{array}{l}
\mathrm{Q}_{1 / \lambda}, \lambda>0, \\
0, \quad \lambda \leq 0,
\end{array} \text { for any } \lambda \in \mathbb{R} .\right.
$$

Note that $E_{0+}=\mathrm{Q}_{\infty}$. Using the change of variable $s=1 / \lambda$, the spectral decomposition of $\mathcal{L}$ in the classical form becomes

$$
\mathcal{L}=\int_{[0,+\infty)} \lambda d E_{\lambda}=-\int_{(0, \infty)} \frac{1}{s} d \mathrm{Q}_{s} .
$$

The $L^{2}$-spectrum of the Laplacian $\mathcal{L}$. For any ball $C \in \mathcal{B}$ define, on the one hand, the function $\phi_{C}$ on $X$ as

$$
\phi_{C}=\frac{1}{\mu(C)} \mathbf{1}_{C}-\frac{1}{\mu(B)} \mathbf{1}_{B}=\left[\frac{1}{\mu(C)}-\frac{1}{\mu(B)}\right] \mathbf{1}_{C}-\frac{1}{\mu(B)} \mathbf{1}_{B \backslash C},
$$

where $B$ is the parent ball of $C$, i.e., $\mathbf{1}_{B}=\mathbf{1}_{C}+\mathbf{1}_{B \backslash C}$. On the other hand, for any $C \in \mathcal{B}$, set also

$$
\lambda(C)=\frac{1}{\operatorname{diam}(C)} .
$$

If $C=X$, then set $\phi_{C} \equiv 1$ and $\lambda(C)=0$. Using the linear independence of the indicators of the child balls (1), one has that, for any parent $B, \sum_{C \prec B} \mu(C) \phi_{C}=$ 0 .

The following result reveals the spectral nature of the pair $\left(\phi_{C}, \lambda(C)\right)$.

Proposition 1. We can see that $\phi_{C}$ is an eigenfunction of $\mathcal{L}$ with the eigenvalue $\lambda(C)$, i.e.,

$$
\mathcal{L} \phi_{C}=\lambda(C) \phi_{C}
$$

Proof. For $C \in \mathcal{B}$ of radius $r=\operatorname{diam}(C)$, where $B$ is the parent one of radius $r^{\prime}$. Any ball of radius $s<r^{\prime}$ either is disjoint with $C$ or is contained in $C$, which implies that $\mathbf{1}_{C}$ is constant in any such ball. It follows that, for any $s<r^{\prime}$, we have $\mathrm{Q}_{s} \mathbf{1}_{C}=\mathbf{1}_{C}$ and, similarly $\mathrm{Q}_{s} \mathbf{1}_{B}=\mathbf{1}_{B}$, whence

$$
\mathrm{Q}_{s} \phi_{C}=\phi_{C}
$$

For $s \geq r^{\prime}$ any ball of radius $s$ either contains both balls $C, B$ or is disjoint from $B$. Since the averages of the two functions $\frac{1}{m(C)} \mathbf{1}_{C}$ and $\frac{1}{m(B)} \mathbf{1}_{B}$ over any ball containing $C$ and $B$ are equal, we obtain that in this case $Q_{s} \phi_{C}=0$. It follows that

$$
\mathcal{L} \phi_{C}=-\int_{(0, \infty)} \frac{1}{s} \mathrm{Q}_{s} \phi_{C} d s=\frac{1}{r^{\prime}} \phi_{C}=\lambda(C) \phi_{C} .
$$


More generaly, we have the following result on the complete representation of Laplacian on an ultrametric space as a base of ball-based eigenfunctions.

Theorem 1 ((Bendikov et al., 2014) [3]). For any $C \in \mathcal{B}$ the function $\phi_{C}$ is an eigenfunction of $\mathcal{L}$ with the eigenvalue $\lambda(C)$. The family $\left\{\phi_{C}: C \in \mathcal{K}\right\}$ is complete in $L^{2}(X, \mu)$. Consequently, the operator $\mathcal{L}$ has a complete system of compactly supported eigenfunctions, called the Haar system associated to $(X, d, \mu)$.

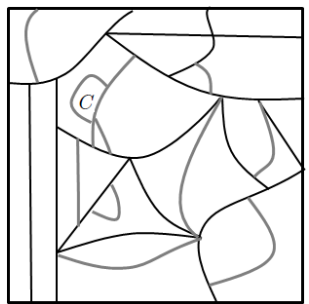

(a)

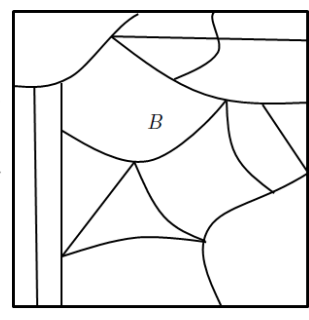

(b)

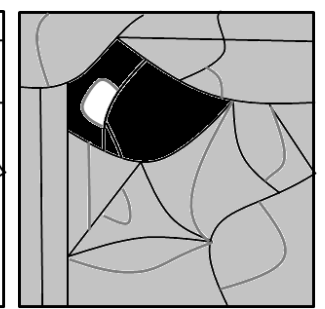

(c)

Fig. 1. Eigenfunction of an ultrametric ball : (a) and (b) depict two partitions of a discrete ultrametric space, where the ball $B$ is the father of ball $C$; (c) eigenfunction of ball $C, \phi_{C}$, in grey, the value of the function is 0 , in white, it is a positive value and in black, a negative one.

Theorem 2 ((Benkikov et al., 2014) [3]). The spectrum $\operatorname{spec} \mathcal{L}$ of the Laplacian $\mathcal{L}$ is given by

$$
\operatorname{spec} \mathcal{L}=\overline{\left\{\frac{1}{r}: r \in \Lambda\right\}} \cup\{0\}, \text { where }\{0\} \text { is for } \lambda(X)=0 .
$$

The hierarchical Laplacian $\mathcal{L}$ on a function. Given the ultrametric measurable set $(X, d, \mu)$, starting from (10), one defines (pointwise) the Laplacian on a function $f \in \mathcal{F}$ as [3]:

$$
\mathcal{L} f(x)=\sum_{C \in \mathcal{B}: x \in B} \kappa(C)\left(f(x)-\frac{1}{\mu(C)} \int_{C} f d \mu\right)
$$

with the positive scaling function given typically by $\kappa(C)=\operatorname{diam}(C)^{-1}-\operatorname{diam}(B)^{-1}$, where $B$ is the parent ball of $C$.

The operator $(\mathcal{L}, \mathcal{F})$ acts in $L^{2}(X, \mu)$, is symmetric, self-adjoint and admits a complete system of eigenfunctions $\left\{\phi_{C}\right\}$ given by (11), where $C \subset B$ run over all nearest neighboring balls in $\mathcal{B}$ [3]. For any two distinct balls $C$ and $C^{\prime}$, the functions $\phi_{C}$ and $\phi_{C^{\prime}}$ are orthogonal in $L^{2}(X, \mu)$. The corresponding eigenvalue 
is given by $\lambda(C)=\operatorname{diam}(C)^{-1}$. Note that one does not need to specify the ultrametric $d$ for the function $\kappa(C)$ and the corresponding eigenvalue $\lambda(C)$. One only requires the family of balls $\mathcal{B}$, or in other words, the intrinsic ultrametric distance $d_{*}(x, y)=\operatorname{diam}(C)$. However, the practical computation of the hierarchical Laplacian (13) requires a sum over all the balls of $\mathcal{B}$.

\section{Laplace operator on a discrete ultrametric space}

Let us now focus on a discrete ultrametric space $(X, d)$. Using the following functional identity (see Introduction) $P=\exp (\mathcal{L})$, whence $\mathcal{L}=\log \frac{1}{P}$, and taking $t=1$ in the discrete heat kernel expression (9), it holds [2]:

$$
\mathcal{L}=\sum_{k=0}^{\infty}\left(\log \frac{1}{s_{k}}\right)\left(Q_{k}-Q_{k+1}\right)=\sum_{k=0}^{\infty} l_{k}\left(Q_{k}-Q_{k+1}\right),
$$

with $l_{k}=-\log s_{k}$, where the series converges in the strong operator topology of $L^{2}(X, m)$. Consequently, $\mathcal{L}$ is a bounded, non-negative definite, self-adjoint operator in $L^{2}(X, m)$, and the discrete spectrum of $\mathcal{L}$ is given by

$$
\operatorname{spec}_{L^{2}} \mathcal{L}=\left\{l_{k}\right\}_{k=0}^{\infty} \cup\{0\} .
$$

Thus each $l_{k}$ is an eigenvalue of $\mathcal{L}$.In particular, the point 0 of $\operatorname{spec}_{L^{\infty}} \mathcal{L}$ is an eigenvalue of multiplicity 1 .

Applied to any $f \in L^{2}(X)$, we have now

$$
\mathcal{L} f=\sum_{k=0}^{\infty} l_{k}\left(Q_{k} f-Q_{k+1} f\right)=l_{0} f-\sum_{k=1}^{\infty}\left(l_{k-1}-l_{k}\right) Q_{k} f .
$$

Here also the pratical interest of this expression of Laplacian of $f$ is obvious, since the basic ingredient is the set of averaging operators $Q_{k}$ on $f$.

Considering the counting measure for $Q_{k}$, discrete ultrametric Laplacian (14) can be written as:

$$
\mathcal{L} f(x)=\alpha\left(f(x)-\frac{1}{\alpha} \sum_{k=1}^{\infty} \frac{\beta_{k}}{\left|B_{k}(x)\right|} \sum_{z \in B_{k}(x)} f(z)\right),
$$

with $\alpha=\log \frac{1}{s_{0}}>0$ and $\beta_{k}=\log \frac{s_{k}}{s_{k-1}}=\log \frac{\sum_{j=0}^{k} c_{j}}{\sum_{j=0}^{k-1} c_{j}}>0$. Up to the minus sign of $\mathcal{L}=-\Delta$, this form (15) can be compared with the discrete Laplacian on $\mathbb{Z}^{n}$ (see Introduction):

$$
\Delta f(x)=-|\widetilde{N}|\left(f(x)-\frac{1}{|\widetilde{N}|} \sum_{z \in \widetilde{N}(x)} f(z)\right) .
$$

If $f$ is a non-negative function on $X$ such that $\mathcal{L} f \equiv 0$, then $f=$ const. 
Deformation of Laplace operator. Let $\mathcal{L}$ be the Laplace operator of the semigroup $\left\{P_{t}\right\}_{t \geq 0}$. Let $\xi:[0, \infty) \rightarrow[0, \infty)$ be a continuous, strictly monotone increasing function with $\xi(0)=0$. Then, the operator $\xi(\mathcal{L})$ is the Laplace operator of the semigroup $\left\{P_{t}^{\xi}\right\}_{t \geq 0}$, defined by [2]

$$
P^{\xi}=\sum_{k=0}^{\infty} c_{k}^{\xi} Q_{k}
$$

where the stochastic sequence of $c_{k}^{\xi}$ is given by $c_{0}^{\xi}=e^{-\xi\left(l_{0}\right)} ; c_{k}^{\xi}=e^{-\xi\left(l_{k}\right)}-$ $e^{-\xi\left(l_{k-1}\right)} ; k \geq 1$.

Example. Taking the deformation $\xi(t)=1-e^{-\alpha t}$, we have:

$$
\mathcal{L}_{\xi}=\xi(\mathcal{L})=\sum_{k=0}^{\infty} \xi\left(l_{k}\right)\left(Q_{k} f-Q_{k+1} f\right) \sum_{k=0}^{\infty}\left(1-s_{k}^{\alpha}\right)\left(Q_{k} f-Q_{k+1} f\right)=\mathrm{id}-P_{\alpha} .
$$

Thus, the residue between $f$ and the heat operator at scale $\alpha, P_{\alpha} f$, can be seen as a Laplacian, "deformation" of the standard one by $1-e^{-\alpha \mathcal{L} f}$.

Ultrametric infinity Laplace operator. Let us first consider the following result.

Theorem 3 ((Benkikov et al., 2012) [2]). For any $p \in[1,+\infty]$, the operator $\mathcal{L}$ can be extended as a bounded operator acting on $L^{p}=L^{p}(X, \mu)$. Moreover, we have

$$
\operatorname{spec}_{L^{p}} \mathcal{L}=\operatorname{spec}_{L^{2}} \mathcal{L}, \text { for any } p \in[1,+\infty] .
$$

Thus, the spectrum of the ultrametric discrete $L^{p}$ Laplacian is the same for any $p$, including the ultrametric infinity Laplacian. This is an important difference with respect to the Euclidean and metric-space cases, where the infinity Laplacian is a very particular operator [8]. Indeed, the infinity Laplacian is a 2nd-order partial differential operator, which in the discrete case of a graph

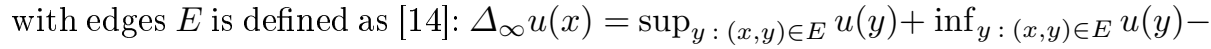
$2 u(x)$, which corresponds just to the defintion of the morphological Laplacian [10] on the graph of edges $E$; i.e., dilation + erosion - 2 function. Inspired by this equivalence, we can propose a morphological inspired infinity Laplacian which mimics the discrete one on a graph by using the notion of (discrete) ultrametric dilation $D_{t}$ and erosion $E_{t}$ introduced in [1]:

$$
\begin{array}{r}
\mathcal{L}_{t}^{\infty} f(x)=2 f(x)-\left(D_{t} f(x)+E_{t} f(x)\right) \\
=2 f(x)-\left(\sup _{0 \leq k \leq \infty}\left\{\sup _{y \in B_{k}(x)} f(y) \wedge b_{k, t}\right\}+\inf _{0 \leq k \leq \infty}\left\{\inf _{y \in B_{k}(x)} f(y) \vee\left(M-b_{k, t}\right)\right\}\right),
\end{array}
$$

where $b_{k, t}=M-t^{-1} c_{k}$ is the so-called discrete structuring function a scale $t$, with $M$ just the maximum value of $f(x)$. Note that the change of sign between $\Delta_{\infty}$ and $\mathcal{L}_{t}^{\infty}$ is only a convention to be consistent with the ultrametric Laplacian $\mathcal{L} f$. This morphological operator can be compared to (15). Note also that the spectral representation of such ultrametric operators requires naturally the study of the eigenvalues and eigenfunctions of morphological operators. 


\section{Application to ultrametric image processing}

For the examples that we consider here, the ultrametric space $(X, d)$ is built from a minimum spanning tree (MST). First, let $G$ be an edge-weighted undirected neighbor graph with points $x \in X$ as vertices and all edge weights as nonnegative values. An MST of $G$ is a spanning tree that connects all the vertices together with the minimal total weighting for its edges, and let $d(x, y)$ be the largest edge weight in the path of the MST between $x$ and $y$. Then the vertices of the graph $G$, with distance measured by $d$ form an ultrametric space. By thresholding the corresponding MST at $k, 0 \leq k \leq K$, a set of partitions is obtained which produces all balls $B_{k}(x)$. In particular, for the case of the discrete images used in the examples, $G$ is a 4-connected pixel neighbor graph and the edge weights are the grey-level difference.

In addition, the sequence used in the examples is just $c_{k}=k / 20$, with $k=$ $1, \cdots, 20$ (ultrametric distances are quantified into $K=20$ levels).

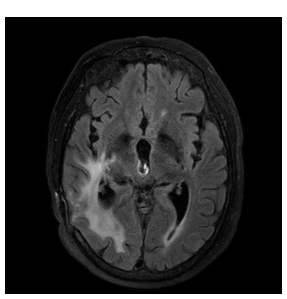

(a)

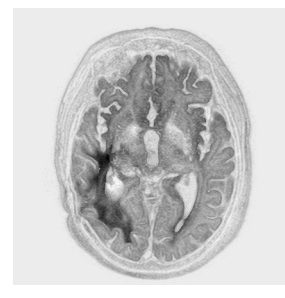

(b)

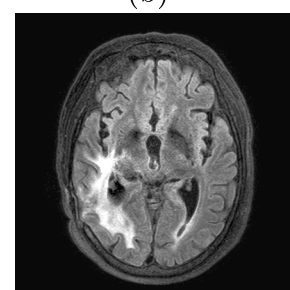

(c)

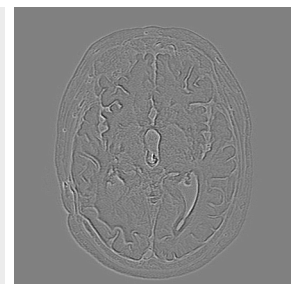

(d)

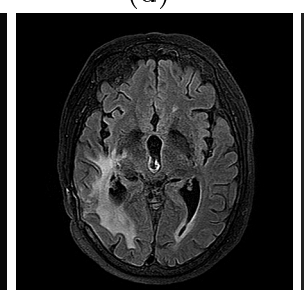

(e)

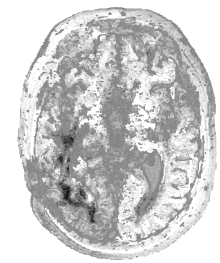

(f)

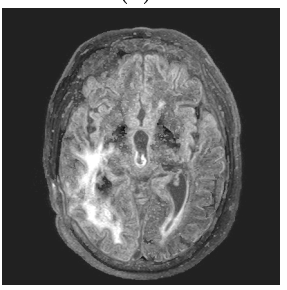

(g)

Fig. 2. Discrete ultrametric Laplace operator: (a) original image $f(x)$, (b) negative of Laplacian $-\mathcal{L} f(x),(\mathrm{c})$ enhanced image by adding the Laplacian $f(x)+\mathcal{L} f(x)$, (d) classical image $\Delta f(x)$, (e) enhanced image by $f(x)-\Delta f(x)$, (f) negative ultrametric morphological Laplacian $-\mathcal{L}_{t}^{\infty} f(x)$, with $t=0.01$ (g) enhanced image by adding morphological Laplacian $f(x)+\mathcal{L}_{t=0.01}^{\infty} f(x)$.

Figure 2 provides an example of the application of discrete Laplace operator on an image endowed with its intrinsic ultrametric distance, which is compared with the standard image Laplacian and with the morphological ultrametric Laplacian, as well as the corresponding enhanced images obtained by adding the Laplacian. From this illustration it can be noted that the enhancement obtained 


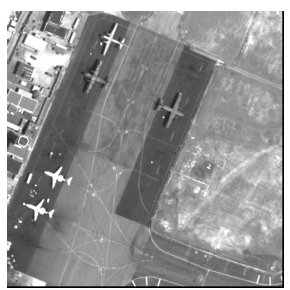

(a)

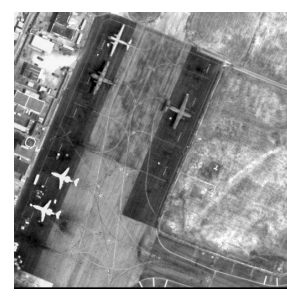

(b)

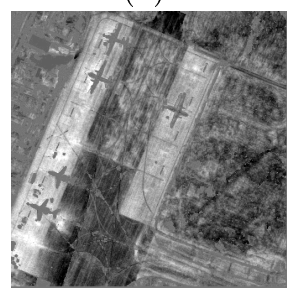

(c)

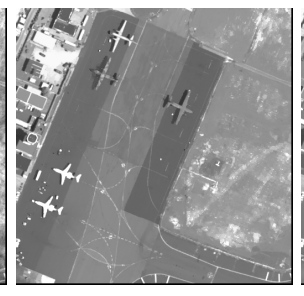

(d)

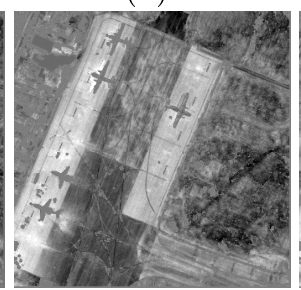

(e)

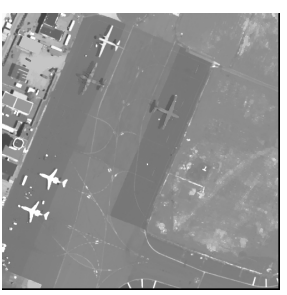

(f)

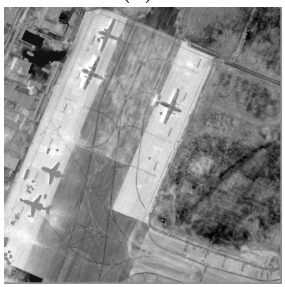

(g)

Fig. 3. Discrete ultrametric Laplace operator and heat kernel: (a) original image $f(x)$, (b) enhanced image by adding the ultrametric Laplacian $f(x)+\mathcal{L} f(x)$, (c) corresponding negative of Laplacian $-\mathcal{L} f(x)$, (d) and (f) ultrametric heat kernel $P_{t} f(x)$, with respectively $t=0.01$ and $t=0.1$, (e) and (g) deformation of Laplace operator corresponding to heat kernel $-\mathcal{L}_{\xi(t)} f(x)$, with respectively $t=0.01$ and $t=0.1$. In residues (e) and (g) the intensities has been stretched.

by the hierarchical Laplace operator is more "regional" than the one by the classical Laplacian. This is expected by the fact that the classical Laplacian is just a second derivative in local neighborhoods. We have observed in similar results from other images that the ultrametric Laplacian improves image dynamics without introducing artifacts. The morphological inspired infinity Laplacian provides a stronger detection of contrasted bright/dark classes of the hierarchy and consequently, the corresponding enhancement is stronger than the ultrametric Laplacian. Note that in the case of the morphological Laplacian, there is a scale parameter $t$, used for the ultrametric multiscale dilation and erosion.

Another comparison is depicted in Figure 3. This time, besides the ultrametric Laplacian and corresponding enhanced image, two examples of the ultrametric heat kernel operator applied on the image are included, with different values of scale parameter $t$. This heat kernel operator provided a smoothing, or ultrametric regularization of the image, similar to a Gaussian filtering, but without any effect of blurring since the underlying ultrametric space contains the hierarchical organization of images zones. The associated deformation of Laplacian (see Section 5) for the heat kernel images are also given, where obviously, the parameter $t$ allows a control of the zones to be detected. 


\section{Conclusions and Perspectives}

The theory reviewed in this paper provides the computational framework to process images or signals defined on a hierarchical representation associated to an ultrametric distance space. In particular, image regularization by ultrametric heat kernel filtering and image enhancement by hierarchical Laplacian have been illustrated. The spectrum of the corresponding Laplacian is just related to the evolution of the size of nested classes along the hierarchy. Our ongoing work will study, on the one hand, the interest of the associated ultrametric pseudodifferential equations [7] in the context of ultrametric images/data processing and on the other hand, the applicative interest of the spectrum of the hierarchical Laplacian to describe shapes and point clouds endowed with an ultrametric structure.

\section{References}

1. J. Angulo, S. Velasco-Forero. Morphological semigroups and scale-spaces on ultrametric spaces. In Proc. of ISMM'17 (13th International Symposium on Mathematical Morphology), LNCS 10225, Springer, pp. 28-39, 2017.

2. A. Bendikov, A. Grigor'yan, Ch. Pittet. On a Class of Markov Semigroups on Discrete Ultra-Metric Spaces, Potential Analysis, 37(2): 125-169, 2012.

3. A. Bendikov, A. Grigor'yan, Ch. Pittet, W. Woess. Isotropic Markov semigroups on ultrametric spaces, Uspekhi Mat. Nauk, 69(4):3-102, 2014.

4. A. Bendikov, P. Krupski. On the spectrum of the hierarchical Laplacian, Potential Analysis, Vol. 41, No. 4, 1247-1266, 2014.

5. A.Bendikov, W.Woess, W.Cygan. Oscillating heat kernels on ultrametric spaces, Journal of Spectral Theory, arXiv:1610.03292, 2017.

6. J. Cousty, L. Najman, Y. Kenmochi, S. Guimarães. Hierarchical segmentations with graphs: quasi-flat zones, minimum spanning trees, and saliency maps, Journal of Mathematical Imaging and Vision, 60(4): 479-502, 2018.

7. A. Khrennikov, S. Kozyrev, W. Zúñiga-Galindo. Ultrametric Pseudodifferential Equations and Applications (Encyclopedia of Mathematics and its Applications). Cambridge University Press, 2018.

8. P. Lindqvist. Notes on the Infinity Laplace Equation, Springer, 2016.

9. D. Marr, E.C. Hildreth. Theory of edge detection. Proc. Roy. Soc. London Seri. B, Vol. 207, 187-217, 1980.

10. L.J. van Vliet, I.T. Young, G.L. Beckers. A Nonlinear Operator as Edge Detector in Noisy Images. Computer Vision, Graphics, and Image Processing, Vol. 45, 167195, 1989.

11. A. Wetzler, Y. Aflalo, A. Dubrovina, R. Kimmel. The Laplace-Beltrami operator: a ubiquitous tool for image and shape processing. In Proc. of ISMM'13 (11th International Symposium on Mathematical Morphology), LNCS 7883, Springer, pp. 302-316, 2013.

12. F. Meyer. Hierarchies of Partitions and Morphological Segmentation. In ScaleSpace and Morphology in Computer Vision, Springer LNCS 2106,161-182, 2001.

13. F. Meyer. Watersheds on weighted graphs, Pattern Recog. Lett., 47:72-79, 2014.

14. Y. Peres, O. Schramm, S. Sheffield, D.B. Wilson. Tug-of-war and the Infinity Laplacian. Journal of the American Mathematical Society, 22(1):167-210, 2009. 